\title{
AN IMBEDDING THEOREM FOR CONNECTED 3-MANIFOLDS WITH BOUNDARY
}

\author{
B. G. CASLER
}

I. Introduction. Suppose $M$ is a compact manifold with a finite triangulation $T$. It is known that, for many algebraic invariants of $T$ (or $M$ ), such as the Euler characteristic, homology, and homotopy, that there is a subcomplex $K$ of $T$ such that $K$ carries all of these algebraic invariants. This subcomplex has recently been called a spine of $M$. This means that one need not consider all of $T$ to consider certain questions usually of an algebraic nature.

While the spine is a useful tool for studying these problems, most of the point-set topological structure of the manifold is lost because of two basic problems of nonuniqueness of spines. The first of these problems is that several manifolds may have the same spine. The second problem is that a given manifold may have several different spines.

In this paper we will explore the first of the problems of nonuniqueness in the case of 3-manifolds. We shall define a type of spine, called a standard spine, and show that (i) each connected compact 3-manifold with boundary has a standard spine and (ii) if two compact 3manifolds have homeomorphic standard spines then the manifolds are homeomorphic.

This means that the standard spine not only carries the algebraic structure but also the point-set topological structure.

Note that a standard spine may not be the simplest-looking spine of a 3-manifold with boundary. For example a circle is the spine of a solid torus and of a solid Klein bottle. It follows that the standard spine of these objects must be more complicated than a circle. In general, each point of a standard spine is contained in a two-dimensional neighborhood homeomorphic to one of three simple-looking sets. Globally, a standard spine may be very complicated, at least, complicated enough to carry orientation. In some cases a standard spine is overcomplicated. For example, a point is a spine of a 3-cell and a 3-cell is the only 3-manifold with boundary that collapses to a point. A point is not a standard spine as a standard spine is a twodimensional object. An example of a standard spine of a 3-cell is shown in Figure 2.

An $n$-manifold, $M$, with boundary is a separable metric space such

Received by the editors March 18, 1964. 
that each point of $M$ is contained in a neighborhood homeomorphic to an $n$-cell. If $p$ is a point of $M$ and $p$ is contained in an open $n$-cell, then $p$ is contained on the interior of $M, \operatorname{Int}(M)$. The boundary of $M$, $\mathrm{Bd}(M)$, is $M-\operatorname{Int}(M)$. If $\operatorname{Bd}(M)$ is empty, then $M$ is an $n$-manifold.

Suppose $K$ is a polyhedron and $\alpha$ is a simplex of $K$ with a face $\beta$. If $\beta$ is the face of no simplex in $K$ except $\alpha$, then we say there is an elementary collapse from $K$ to $K-(\operatorname{Int}(\alpha)+(\operatorname{Int}(\beta))$, where $\operatorname{Int}(\alpha)$ and $\operatorname{Int}(\beta)$ are, respectively, $\alpha-($ faces of $\alpha)$ and $\beta-($ faces of $\beta)$. If $K$ is a polyhedron, $L$ is a subpolyhedron of $K$ and there are polyhedra $K=K_{0} \supset K_{1} \supset \cdots \supset K_{n}=L$ such that there is an elementary collapse from $K_{i-1}$ to $K_{i}, i=1,2, \cdots, n$, then we say $K$ collapses to $L$ [3], [5]. If $K$ is a polyhedron, if $K$ collapses to $L$, and if there is no elementary collapse of $L$, then $L$ is a spine of $K$.

Suppose $M$ is a polyhedron, and $L$ and $K$ are subpolyhedra of $M$. Then a regular neighborhood of $L$ with respect to $K$ is the union of all simplices in $K$ that intersect $L$. We denote the regular neighborhood of $L$ with respect to $K$ as $\eta(L, K)$. Note that, under this definition, $K$ does not have to contain $L$.

Suppose $K$ is a polyhedron. Then the singular $n$-skeleton, $K_{n}$, of $K$ is the set of points in $K$ such that if $p \in K_{n}$, then no neighborhood of $p$, open in $K$, is homeomorphic to Euclidean $(n+1)$-space.

Let the set $A$ be the join of the point $(0,0,1)$ in $E^{3}$ and the line segment $[(1,0,0)(-1,0,0)]$, the set $B$ the join of the point $(1,0,0)$ and the line segment $[(0,1,0)(0,-1,0)]$, the set $C$ the join of the point $(-1,0,0)$ and the line segment $[(0,1,0)(0,-1,0)]$, and the set $D$ be the join of the point $(0,0,-1)$ and the line segment $[(0,1,0)(0,-1,0)]$. Let a standard neighborhood of $T y p e \mathrm{I}$ be $B+C$, a standard neighborhood of Type II be $A+B+C$ and $a$ standard neighborhood of Type III be $A+B+C+D$, as shown in Figure 1.

Suppose $K$ is a 2-dimensional polyhedron, $K_{1}$ is the singular 1skeleton of $K$, and $K_{0}$ is the singular 0 -skeleton of $K_{1}$. The polyhedron $K$ is a standard 2-polyhedron if each point of $K$ is contained in a set open in $K$ whose closure is homeomorphic to one of the three standard neighborhoods, if $K_{1}-K_{0}$ is the sum of a countable number of pairwise disjoint open arcs, and if $K-K_{1}$ is the sum of a countable number of disjoint open disks. If $K$ is a standard 2-polyhedron and if $K$ is the spine of a connected manifold $M$ with nonvoid boundary, then $K$ is a standard spine of $M$.

It is known that every connected 3-manifold with nonvoid boundary has a standard spine. However, a proof of this fact has not appeared in print. Therefore, to complete the paper we will prove this theorem in $\S I V$. 


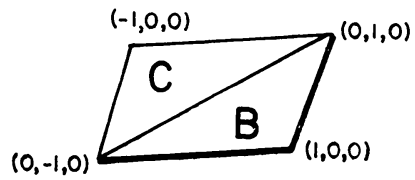

\section{Type I}



Type II

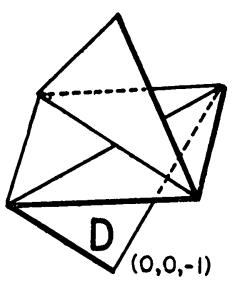

Type III

FIGURE 1

II. The imbedding theorem. We can now state and prove the main result of this paper.

Theorem 1. Suppose $M$ is a compact connected 3-manifold with nonvoid boundary and $K$ is a standard spine of $M$ such that $K$ can be polyhedrally imbedded in the interior of a 3-manifold with boundary, $N$. Then the imbedding of $K$ can be extended to imbed $M$ in $N$.

Proof. Let $M$ be a compact connected 3-manifold with nonvoid boundary. As Moise [2] and Bing [1] have shown that every 3manifold can be triangulated, we may assume that $M$ is triangulated. Let $K$ be a standard spine of $M$ contained on the interior of $M$. Let $T$ be a finite triangulation of $M$ such that $K$ is a full subpolyhedron of $M$, that is, if all of the vertices of a simplex $\alpha$ are contained in $K$, then $\alpha$ is contained in $K$. Let $K_{1}$ be the singular 1-skeleton of $K$ and $K_{0}$ the singular 0 -skeleton of $K_{1}$. If $v_{i}$ and $v_{j}$ are distinct vertices of $K_{0}$, we may assume that $T$ is fine enough that $\eta\left(v_{i}, M\right)$ and $\eta\left(v_{j}, M\right)$ are disjoint closed sets.

Let $\gamma$ be a piecewise linear homeomorphism of $K$ into $N$, an orientable 3-manifold with boundary. Let $\gamma(K)=L$, and let $L_{1}$ be the singular 1-skeleton of $L$, and $L_{0}$ the singular 0 -skeleton of $L_{1}$. Let $T^{\prime}$ be a triangulation of $N$ such that $L$ is contained as a polyhedron in the 2-skeleton of $N, L$ and $L_{1}$ are full subpolyhedra of $N$, and $\gamma^{-1}\left(\eta\left(L_{0}, L\right)\right)$ is contained in the interior of $\eta\left(K_{0}, M\right)$. 
We shall now extend $\gamma$ to a homeomorphism, $\phi$, from $\eta(K, M)$ into $N$.

Step 1. Suppose $v$ is a 0 -simplex in $K_{0}$ and $u=\gamma(v)$ is a 0 -simplex in $L_{0}$. Let $\eta(v, M)=C$ and $\eta(u, N)=C^{\prime}$. In this step we shall describe a homeomorphism between $C$ and $C^{\prime}$.

We may consider $C$ (and also $C^{\prime}$ ) as a tetrahedron in the following way. Let $K_{1} \cdot \operatorname{Bd}(C)$ be the vertices of $C$. This is possible, as exactly four 1-simplices of $K_{1}$ intersect in $v$. As exactly three 2-simplices of $K$ intersect in each 1 -simplex of $K_{1}, \operatorname{Bd}(C) \cdot\left(K-K_{1}\right)$ is the sum of exactly six open arcs. It follows that $K \cdot \operatorname{Bd}(C)$ is homeomorphic to the 1-skeleton of a tetrahedron. If there is a homeomorphism of the vertices of one tetrahedron on to the vertices of another tetrahedron, then this homeomorphism may be extended to the 1-skeleton, then to the 2-skeleton and, finally, to the tetrahedra.

Let $e_{1}, e_{2}, e_{3}$, and $e_{4}$ be the four 1-simplices of $K^{1}$ that intersect at $v$. Let $\phi\left(e_{i} \cdot \operatorname{Bd}(C)\right)$ be defined so that $\gamma^{-1} \phi\left(e_{i} \cdot \operatorname{Bd}(C)\right)$ is contained in $e_{i}, i=1,2,3,4$. This may be done as $\gamma^{-1}\left(\eta\left(L_{0}, L\right)\right.$ is contained on the interior of $\eta\left(K_{0}, M\right)$. This defines $\phi$ to take $K_{1} \cdot \operatorname{Bd}(C)$ on to $L_{1} \cdot \operatorname{Bd}\left(C^{\prime}\right)$, that is, the vertices of one tetrahedron onto the vertices of another. It follows that $\phi$ may be extended to take $C$ onto $C^{\prime}$ in such a way that

$$
\phi \mid \eta\left(K_{1}, \mathrm{Bd}(C)\right)=\eta\left(L_{1}, \mathrm{Bd}\left(C^{\prime}\right)\right)
$$

and

$$
\phi \mid \eta(K, \mathrm{Bd}(C))=\eta\left(L, \operatorname{Bd}\left(C^{\prime}\right)\right) .
$$

We complete Step 1 by defining this homeomorphism on the regular neighborhood of each vertex in $K_{0}$. Hence, $\phi\left(\eta\left(K_{0}, M\right)\right)=\eta\left(L_{0}, N\right)$.

Step 2. Let $F$ be the closure of a component of $\eta\left(K_{1}, M\right)-\eta\left(K_{0}, M\right)$. As each component of $K_{1}-K_{0}$ is an open arc, $F$ is a 3 -cell. Further, $F \cdot\left(\eta\left(K_{0}, M\right)\right)$ is the sum of two disks $D_{1}$ and $D_{2}$. As each 1-simplex of $K_{1}$ is contained in exactly three 2 -simplices of $K, K \cdot(\operatorname{Bd}(F)$ $\left.-\left(D_{1}+D_{2}\right)\right)$ is the sum of three open $\operatorname{arcs} A_{1}, A_{2}$, and $A_{3}$. The closure of each of these open arcs contains a point of $D_{1}$ and a point of $D_{2}$. Further, $\operatorname{Bd}(F)-\left(\sum D_{j}+\sum A_{i}\right), j=1,2, i=1,2,3$, is the sum of exactly three open disks.

By Step 1 we find that one component of the closure of $\eta\left(L_{1}, N\right)$ $-\eta\left(L_{0}, N\right)$ will contain $\phi\left(D_{1}+D_{2}\right)$. Let $F^{\prime}$ be this component. Let $\phi\left(D_{j}\right)=D_{j}^{\prime}, j=1,2$. As $L$ is homeomorphic to $K, L \cdot\left(\operatorname{Bd}\left(F^{\prime}\right)-\sum D_{j}^{\prime}\right)$ is the sum of three open $\operatorname{arcs} A_{1}^{\prime}, A_{2}^{\prime}$, and $A_{3}^{\prime}$. As $\phi$ is defined on the endpoints of the $A_{i}^{\prime}, \phi$ may be extended to take $A_{i}$ onto $A_{i}^{\prime}$. As $\operatorname{Bd}\left(F^{\prime}\right)-\left(\sum D_{j}^{\prime}+\sum A_{i}^{\prime}\right)$ is the sum of exactly three open disks, $\phi$ 
may be extended to take $\operatorname{Bd}(F)$ onto $\operatorname{Bd}\left(F^{\prime}\right)$ in such a way that $\phi \mid \eta(K, \operatorname{Bd}(F))=\eta\left(L, \operatorname{Bd}\left(F^{\prime}\right)\right)$, and, hence, $\phi$ may be extended to take $F$ onto $F^{\prime}$. We complete Step 2 by defining this homeomorphism on each component of the closure of $\eta\left(K_{1}, M\right)-\eta\left(K_{0}, M\right)$. Hence, $\phi\left(\eta\left(K_{1}, M\right)=\eta\left(L_{1}, N\right)\right)$.

Step 3. As all "nonmanifold" points of $K$ are contained in $K_{1}$, the closure of $K-\eta\left(K_{1}, M\right)$ is a 2-manifold with boundary. As each component of $K-K_{1}$ is an open disk, the closure of each component of $K-\eta\left(K_{1}, M\right)$ is a disk. In the same way the closure of each component of $L-\eta\left(L_{1}, M\right)$ is a disk.

Let $P$ be a component of the closure of $K-\eta\left(K_{1}, M\right)$. Let $P^{\prime}$ be the component of the closure of $L-\eta\left(L_{1}, N\right)$ intersected by $\gamma(P)$. As $P$ and $P^{\prime}$ are homeomorphic and $\phi$ is defined to take $\operatorname{Bd}(P)$ on to $\operatorname{Bd}\left(P^{\prime}\right)$, $\phi$ may be extended to take $P$ onto $P^{\prime}$. As $K$ and $L$ are full subpolyhedra, and as $P$ and $P^{\prime}$ are disks, the closure of $\eta(P, M)-\eta\left(K_{1}, M\right)$ is a 3 -cell and, hence, may be expressed as $P \times I$ where $P \times[1 / 2]=P$, and the closure of $\eta\left(P^{\prime}, N\right)-\eta\left(L_{1}, N\right)$ may be expressed as $P^{\prime} \times I$ where $P^{\prime} \times[1 / 2]=P^{\prime}$. It follows that $(P \times I) \cdot\left(\eta\left(K_{1}, M\right)\right)=\operatorname{Bd}(P)$ $X I$. Hence, $\phi(\operatorname{Bd}(P) \times I)$ is defined and equal to $\operatorname{Bd}\left(P^{\prime}\right) \times I$. It follows that $\phi$ may be extended to take $P \times I$ onto $P^{\prime} \times I$. In the same way, $\phi$ may be extended to take $\eta(K, M)$ onto $\eta(L, N)$.

We complete the proof of this theorem by noting that there is a homeomorphism $h$ from $M$ onto $\eta(K, M)$ keeping $K$ fixed [4], [6] and, hence, the composition $\phi h$ is an imbedding of $M$ in $N$ extending the given imbedding $\gamma$ of $K$ in $N$.

We obtain the following two corollaries from Theorem 1.

Corollary 1. If $K$ is a standard spine of a compact 3-manifold with nonvoid boundary $M$ and $K^{\prime}$ is a standard spine of a compact 3-manifold with nonvoid boundary $M^{\prime}$ and $K$ is homeomorphic to $K^{\prime}$, then $M$ is homeomorphic to $M^{\prime}$.

I wish to thank J. J. Andrews for pointing out the above corollary.

CoRollary 2. Suppose $M$ is a contractible compact 3-manifold with nonvoid boundary and $K$ is a standard spine of $M$ such that $K$ can be imbedded in $E^{3}$. Then $M$ can be imbedded in $E^{3}$ and $M$ is a 3-cell.

III. Bing's house with two rooms. There are many known spines of a 3-cell. The following example, due to R. H. Bing, has some useful properties, one of which we will use in proving Theorem 2 in $\S I V$.

The most instructive method of describing this example is to show a picture of this 2-polyhedron. This we have done in Figure 2. We see in Figure 2 that $T$ is a square disk with an open circular disk re- 


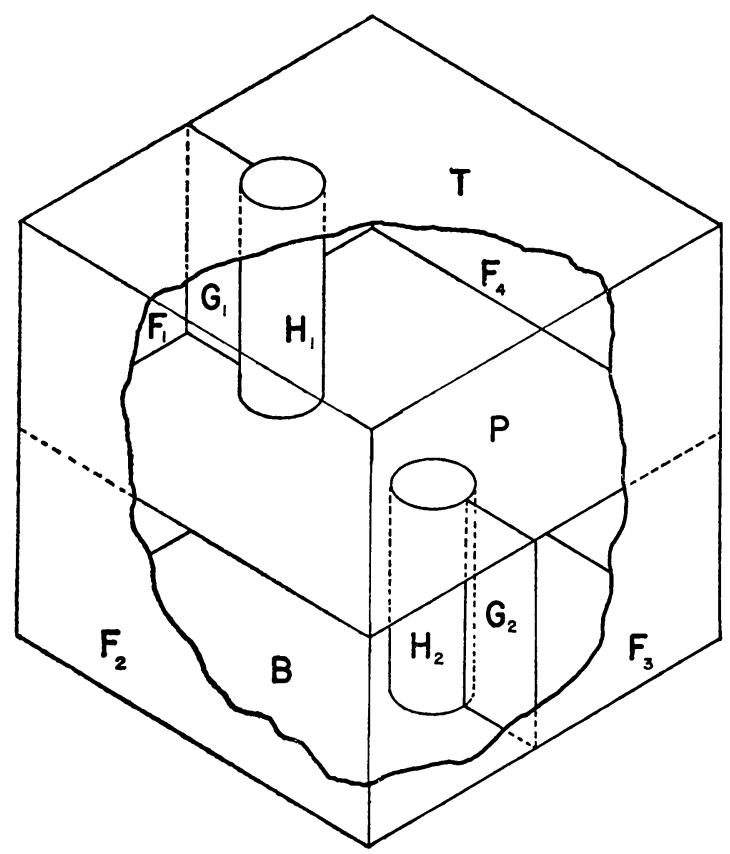

FIGURE 2

moved, $P$ is a square disk with two open circular disks removed, $B$ is a square disk with an open circular disk removed, $F_{1}, F_{2}, F_{3}$, and $F_{4}$ are square disks, $H_{1}$ and $H_{2}$ are right circular cylinders, and $G_{1}$ and $G_{2}$ are rectangular disks. Let this polyhedron be called $X$. This polyhedron $X$ is named Bing's house with two rooms.

The property that we will use in $\S I V$ is the following. Let $C$ be a cube. Then there is a collapse of $C$ on to $X$ such that $\operatorname{Bd}(C)-X$ is the sum of two open disks. Let the closure of these disks be called $D_{1}$ and $D_{2}$. As $X$ is a standard 2-polyhedron, $X$ is a standard spine of $C$. Let $X_{1}$ be the singular 1-skeleton of $X$ and $X_{0}$ the singular 0 -skeleton of $X_{1}$. Let $J=\operatorname{Bd}(C) \cdot P$ and $A_{i}=\operatorname{Bd}(C) \cdot G_{i}, i=1,2$.

Suppose $M$ is a 3-manifold with nonvoid boundary and $T$ is a triangulation of $M$. Let $K$ be a spine of $M$ in the triangulation $T$. Let $Q$ be a 3 -cell in $M$ intersecting $K$ such that $K \cdot \operatorname{Bd}(Q)$ is at most a 1dimensional set and that $M$ collapses to $Q+K$. Choose any 2 -simplex, $\alpha$, in $\operatorname{Bd}(Q)$. There is a piecewise linear homeomorphism, $\gamma$, from $C$ on to $Q$ such that $\gamma\left(D_{1}+A_{1}+A_{2}+J\right)$ is contained in the interior of $\alpha$. As $K$ intersects $\mathrm{Bd}(Q)$ in at most a 1 -dimensional set, $K-\operatorname{Int}(Q)$ does not intersect $\gamma\left(X_{1}\right)+\gamma\left(D_{1}+D_{2}\right)$. As $\gamma\left(D_{1}+D_{2}\right)$ does not inter- 
sect $K-\operatorname{Int}(Q), K+Q$ collapses onto $(K-\operatorname{Int}(Q))+\gamma(X)=K^{\prime}$. It follows that $K^{\prime}$ is a spine of $M$. Further, as $K-\operatorname{Int}(Q)$ does not intersect $\gamma\left(X_{1}\right)$, each point of $\gamma\left(X_{1}\right)$ is contained in a set open in $K^{\prime}$ whose closure is homeomorphic to a standard neighborhood of Type II or Type III.

IV. The construction of a standard spine. We will now give the construction of a standard spine in the proof or the next theorem.

Theorem 2. If $M$ is a compact connected 3-manifold with nonvoid boundary, then $M$ has a standard spine.

Proof. Let $K^{i}, i=0,1,2$, be the $i$-skeleton of any spine $K$ of $M$, contained in $\operatorname{Int}(M)$. We note that $K^{i}$ is the entire $i$-skeleton of $K$ as distinct from the singular $i$-skeleton, $K_{i}$. Let $v_{1}, v_{2}, \cdots, v_{i}, \cdots$ be the elements of $K^{0}, e_{1}, e_{2}, \cdots, e_{j}, \cdots$ be the elements of $K^{1}$. Let $V_{i}=\eta\left(v_{i}, M\right)$ with respect to the second barycentric subdivision of $M$, and $E_{i}$ the closure of $\eta\left(e_{i}, M\right)-\sum V_{i}$ with respect to the third barycentric subdivision of $M$.

It is clear that $M$ collapses to $K+\sum V_{i}+\sum E_{i}$. It is also clear that, for each $V_{i}$, there is a homeomorphism of Bing's house with two rooms into $V_{i}$, as described in \$III. Let $X_{i}$ be the image of Bing's house with two rooms in $V_{i}$. Further, $M$ collapses to $K-\sum V_{i}$ $+\sum X_{i}+\sum E_{i}$. Further, each $E_{i}$ is a cell intersecting $\sum X_{i}$ in the sum of two disks, $D_{i, 1}$ and $D_{i, 2}$.

We may think of $D_{i, 1}$ as a free edge of $E_{i}$. Hence $M$ collapses to $K-\left(\sum V_{i}+\sum E_{i}\right)+\sum X_{i}+\left(\operatorname{Bd}\left(E_{i}\right)-\sum D_{i, 1}\right)=L$. If one looks at the local geometry one will see that each point is contained in a standard neighborhood of Types I, II or III.

Let $L_{i}, i=0,1$, be the singular $i$-skeleton of $L$. To see that $L-L_{1}$ is the union of a finite number of sets, each homeomorphic to an open disk with a finite number (possibly zero) of closed disks removed, note the following four items: (i) Bing's house with two rooms with the singular 1-skeleton removed is the union of three open disks; (ii) $\operatorname{Bd}\left(E_{i}\right)-D_{i, 1}$ is an open disk; (iii) $K-\left(\sum V_{i}+\sum E_{j}\right)$ is the union of open disks; and (iv) $L$ contains a finite number of simplices.

As $L-L_{1}$ is the union of a finite number of open disks with a finite number of closed disks removed, there is a finite number of arcs $A_{i}, i=1,2, \cdots, q$ in $L$ such that each component of $L-\left(L_{1}+\sum A_{i}\right)$ is an open disk.

Further we may choose these arcs in such a way that $L_{0} \cdot \sum A_{i}=\varnothing$. It follows that we may take a regular neighborhood $N_{i}$ about $A_{i}$ for each $i$ such that $N_{i} \cdot N_{j}=\varnothing$ for $i \neq j$ and $N_{i}-L$ is the union of four 
components, the closure of each of which is a cell. As $N_{i}$ is the regular neighborhood of an arc, it follows that $N_{i}$ is a cell. Further, $L-\left(L_{1}+\sum N_{i}\right)$ is the union of disjoint open disks. We obtain the desired spine by collapsing the $N_{i}$ from one of the two of $L-N$ : whose closure does not contain $\operatorname{Int}\left(A_{i}\right)$.

V. Questions. It would be interesting to know whether a theorem of this general nature would hold for higher-dimensional manifolds. One problem would be the identification of the proper standard neighborhoods. A step in this direction is the observation that the dual 2-skeleton of a 3-manifold have the proper standard neighborhood.

In another direction it would be of interest to see if there is any way to associate a unique standard spine with a given manifold.

\section{REFERENCES}

1. R. H. Bing, An alternative proof that 3-manifolds can be triangulated, Ann. of Math. (2) 69 (1959), 37-65.

2. E. E. Moise, Affine structures in 3-manifolds. V. The triangulation theorem and Hauptvermutung, Ann of Math. (2) 56 (1952), 96-114.

3. J. H. C. Whitehead, Simplicial spaces, nuclei, and m-groups, Proc. London Math. Soc., 45 (1939), 243-327.

4. - The immersion of an open 3-manifold in Euclidean 3-space, Proc. London Math. Soc. (3) 11 (1961), 81-90.

5. E. C. Zeeman, Polyhedral n-manifolds. II. Embeddings, Topology of 3-manifolds and related topics, pp. 64-70, Prentice-Hall, Englewood Cliffs, N. J., 1962.

6. J. F. P. Hudson and E. C. Zeeman, On regular neighbourhoods, Proc. London Math. Soc. (3) 14 (1964), 719-745.

Elouisiana State University 